\title{
COMPARISON OF VARIOUS NOISE MITIGATION TECHNIQUE USED WITH CLIPPING FOR REDUCTION OF PAPR IN OFDM
}

\author{
Hemant Choubey ${ }^{1}$, Aparna Singh ${ }^{2}$, Amit Shukla ${ }^{3}$ \\ ${ }^{1,3}$ Student, ${ }^{2}$ Asst Professor, Electronics and Communication, UIT, M.P, India \\ hemantchoubey271986@gmail.com,aparna.kushwah@gmail.com,amit.rgi@gmail.com
}

\begin{abstract}
A simple technique used to reduce the PAPR of OFDM signals is to clip the signal to a maximum allowed value, at the cost of BER degradation and out-of-band radiation. Clipping does not add extra information to the signal and high peaks occur with low probability so the signal is seldom distorted. Out-of-band radiation can be reduced by filtering at the transmitter, the filter used in this project consists on a FFT-IFFT pair which is easier to implement than traditional FIR filters and allows the implementation of the clip \& filter set several times in order to reduce the peak re growth that filtering introduces. The BER degradation can be mitigated by reconstructing the signal at the receiver. We analyzed the performance of the decision-aided reconstruction (DAR) and improved DAR (IDAR) techniques that iteratively try to guess the original symbols and proposed an improvement for one of those techniques.
\end{abstract}

Index Terms: Complementary cumulative distribution function (CCDF), high power amplifier (HPA), Orthogonal Frequency Division Multiplexing (OFDM), Peak-to-Average Power Ratio (PAPR), inter-symbol interference (ISI)

\section{INTRODUCTION}

Introducing a filter after the clipping operation helps mitigating the out-of-band radiation introduced by clipping. Filtering, however, distorts also the signal and causes peak re growth [6]. A common filter used for this purpose is the FIR filter. In this application the filter used is the FFT-IFFT filter proposed by Armstrong in [1]. This filter achieves better performance than the FIR filter, it introduces less noise into the signal, causes less peak regrowth and needs less computation; since it operates symbol by symbol it causes no inter-symbol interference. Clipping too large peaks is a simple solution to the PAPR problem. Clipping belongs to the group of techniques that reduce large peaks by nonlinearly distorting the signal [8]. It does not add extra information to the signal and too large peaks occur with low probability so the signal is seldom distorted. The maximum peak power allowed is determined by the system specifications, usually by the linear region of the power amplifier. A maximum peak amplitude $A$ is chosen so that the OFDM signal does not exceed the limits of this region, symbols that exceed this maximum amplitude, will be clipped. The clipping function is performed in digital time domain, before the D/A conversion as shown in Figure 1 and the process is described by the following expression

$$
x_{k}^{z}=\left\{\begin{array}{cc}
x_{k}, & \left|x_{k}\right| \leq A \\
A e^{j \phi\left(x_{k}\right)},\left|x_{k}\right|>A &
\end{array}\right.
$$

Where $\mathrm{x}_{\mathrm{ck}}$ is the clipped signal, $\mathrm{x}_{\mathrm{k}}$ is the transmitted signal, $\mathrm{A}$ is the clipping amplitude and $\mathrm{f}\left(\mathrm{x}_{\mathrm{k}}\right)$ is the phase of the transmitted signal $x_{k}$. The graphical expression of this function is shown in Figure 2. The clipping ratio (CR) is defined as

$$
\text { C.R }=\frac{A}{\sigma}
$$

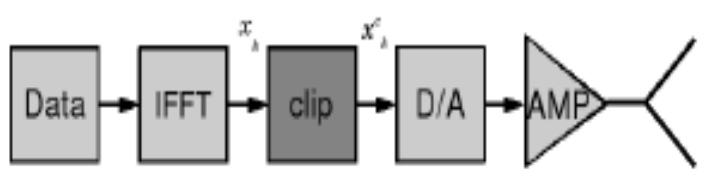

Fig1.Clipping in the transmitter

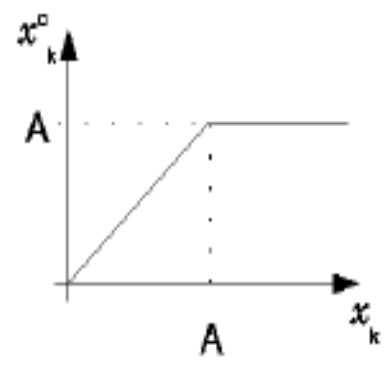

Fig2.Clipping Function 
Clipping is a non-linear process so it introduces in-band distortion (Figure 3), also called clipping noise, and out-ofband radiation and inter-carrier interference (Figure 4), which degrade the system performance and the spectral efficiency. The clipping noise is related to the difference between the original signal $\mathrm{x}_{\mathrm{k}}$ and the clipped signal $x_{k}^{e}$. The signal sent to the receiver is the clipped signal, which is different from the signal that we actually wanted to send. This difference is measured by the signal to clipping noise ratio (SCNR)

$$
\operatorname{SCNR}=\frac{\left|x_{k}\right|^{2}}{\left|x_{k}-x_{k}\right|^{2}}
$$

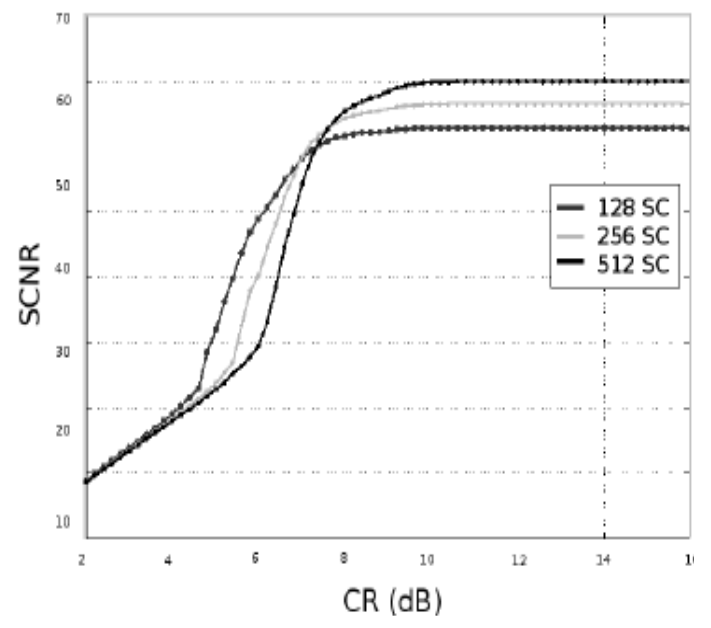

Fig3.SCNR measured for different number of subcarrier

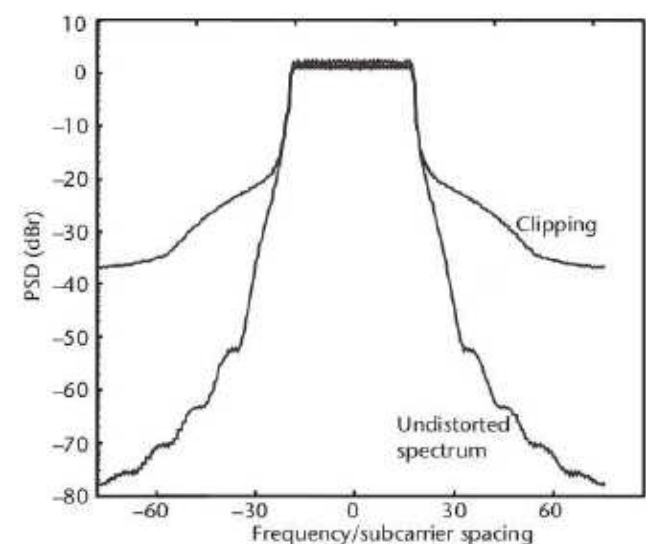

Fig4.Out of band radiation

\section{NOISE MITIGATION}

After clipping the signal, the out-of-band radiation caused by clipping falls in the zeros and then the signal is passed to the FFT filter. The FFT function transforms the clipped signal $x_{k}^{e}$ to frequency domain yielding ${ }^{\mathrm{e}}$. The information components of $\mathrm{X}_{\mathrm{r}}^{\mathrm{c}}$ are passed unchanged to the IFFT block and the out-ofband radiation that fell in the zeros is set back to zero. The IFFT block of the filter transforms the signal to time domain and the obtained signal $\mathrm{x}_{\mathrm{k}}^{\mathrm{F}}$ is passed to the D/A converter. After filtering, the signal suffers peak regrowth so in order to minimize this effect the clip \& filter process can be repeated several times.

\section{NOISE MITIGATION METHODS}

\subsection{Decision-Aided Reconstruction (DAR) Method}

The goal of the clipping operation is to reduce the PAPR of the original signal $\mathrm{x}_{\mathrm{k}}$; it introduces, however, some distortion that makes the recovering of $x_{k}$ in the receiver more difficult. The DAR method was proposed by Kim and Stuber in [5] in order to mitigate the effects of the distortion introduced by clipping [5].It is a nonlinear iterative reconstruction technique implemented in the receiver. To implement this technique, the receiver needs to know the clipping ratio $A$ used at the transmitter. The signal sent by the transmitter in frequency domain can be expressed as

$$
Y_{n}=a_{n} X_{n}+C_{n}
$$

Where $\alpha_{\mathrm{n}}$ is the clipping distortion, which changes randomly from block to block and $\mathrm{C}_{\mathrm{n}}$ are the noise introduced by lipping. The received signal is therefore

$\mathrm{Zn}=\mathrm{h}_{\mathrm{n}} \mathrm{Y}_{\mathrm{n}}+\mathrm{W}_{\mathrm{n}}=\mathrm{h}_{\mathrm{n}} \mathrm{a}_{\mathrm{n}} \mathrm{X}_{\mathrm{n}}+\mathrm{Q}_{\mathrm{n}}$

Where $h_{n}$ is the complex channel gain, that can be accurately estimated, and $Q_{n}$ is the sum of the additive white Gaussian noise (AWGN) and the clipping noise $C_{n}$. In all the simulations performed in this project ideal channel estimation is supposed. Figure 5 shows the block diagram of the DAR method, where $r_{k}$ is the received signal $Z_{n}$ in time domain.

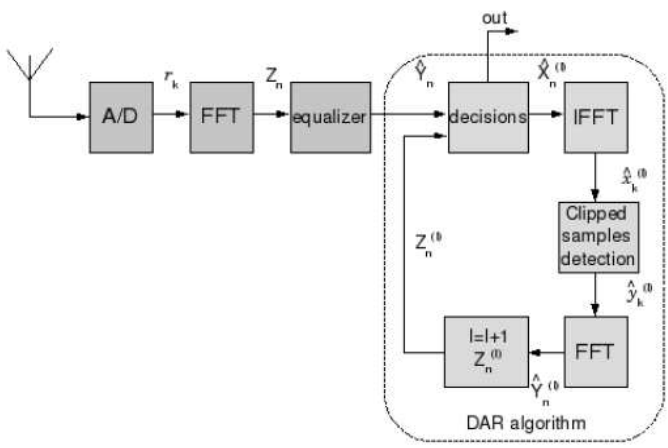

Fig5. DAR algorithm block diagram 
If the CR is too low (this depends on the modulation), the performance of DAR is degraded because false detection increases.

\subsection{Improved Decision-Aided Reconstruction (IDAR)}

\section{Method}

The DAR method was proposed to mitigate the clipping noise but it does not deal with the intermodulation noise. The intermodulation products are reduced by setting the IBO of the power amplifier at the linear region; however, in order to maximize the power efficiency of the amplifier, the IBO is set nearly at the saturation region degrading this way the BER due to the high non-linearity. The improved DAR method mitigates both the clipping noise and the inter-modulation noise. The IDAR method [2], like the DAR one, is an iterative method applied at the receiver and was proposed by Boonsrimuang et al. in [2], Figure 6 shows the block diagram. It repeats at the receiver the clipping and the amplifying operations, so the receiver needs to know the clipping amplitude A and the amplifier characteristics. In Figure 6 can be seen that the method starts by estimating the original signal like in the DAR method

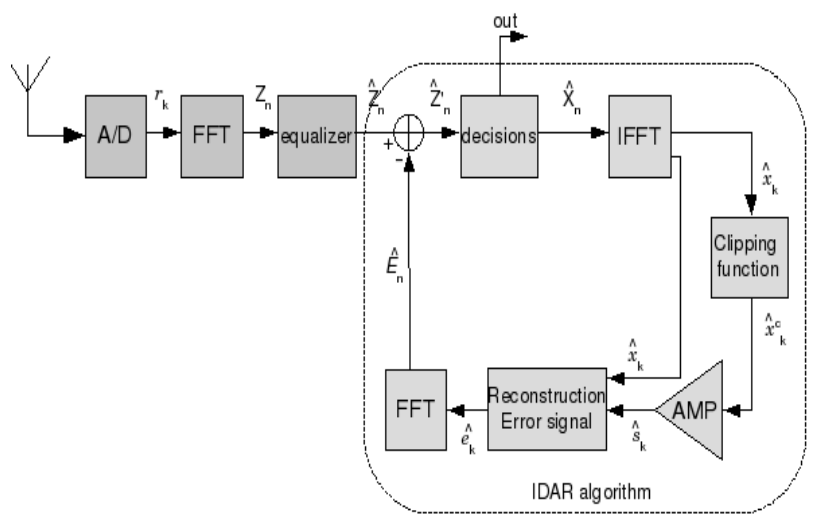

Fig6. IDAR algorithm block diagram

By using this method the intermodulation noise is mitigated, relatively even when the nonlinear amplifier is operated at the saturation region, while with DAR the BER is severely affected by this noise. Although the method shows a good performance for different required signal to noise ratios, for CR lower than $3 \mathrm{~dB}$ around $0 \mathrm{~dB}$.

\subsection{IDAR Including the FFT-IFFT Filter (IDARF)}

The IDAR method shows a very good performance by mitigating the clipping and the intermodulation noises. However, in order to reduce the out-of-band radiation it is advisable to use a filter in the transmitter; in this case the filter used is the FFT-IFFT filter. So, if the clipping operation is followed by the filter at the transmitter, this filter should also be included in the IDAR method at the receiver, Figure7 shows a block diagram of the new method. In order to include the FFT filter in the IDARF method, the same number of zeros as in the transmitter are inserted into the estimated signal before converting it to time domain. Clip \&filter is applied to the time domain signal which is then amplified like in the IDAR method. The zeros are removed from the signal after the amplifier; the signal is transformed to frequency domain, the zeros are removed and the signal is transformed back to time domain in order to calculate the error signal. The error signal is the difference between the estimated signal $\widehat{x}_{k}$ (before adding the zeros) and the signal that suffered the clip\&filter and amplifying process, ${ }^{S_{k}}$, after removing the zeros. The red boxes in Figure 7 show the operations that have to be added to the IDAR algorithm in order to include the FFT filter in the method.

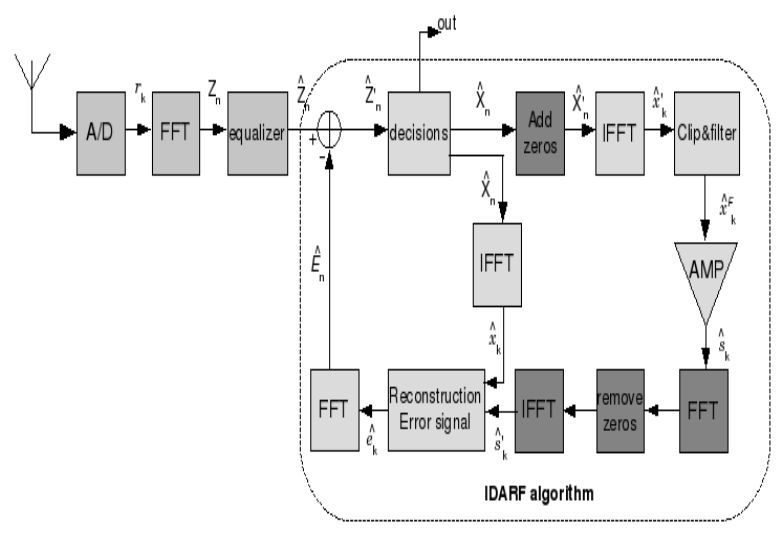

Fig7. IDARF algorithm block diagram

\section{SIMULATION RESULTS}

After theoretically studying noise mitigation techniques, these techniques have been tested both in a flat fading channel and in a selective fading channel. The simulations have been made for the 16-QAM and the 64-QAM modulations and all them use 256 subcarriers, when analyzing the system for different $\mathrm{CR}$, the SNR used varies depending on the modulation, this information is available in Table 3 . The frequency selective channel parameters are shown in Table 1 and the number of iterations used for each method is shown in Table 2.

Table 1: Channel parameters

\begin{tabular}{|l|l|l|}
\hline tap & delay & tap coeff \\
\hline h0 & 0.0 & 0.8405 \\
\hline h1 & 0.3 & 0.4726 \\
\hline h2 & 1.0 & 0.2658 \\
\hline
\end{tabular}


Table 2: number of iterations

\begin{tabular}{|l|l|}
\hline tap & delay \\
\hline clip\&filter & 1 \\
\hline DAR & 2 \\
\hline IDAR & 2 \\
\hline IDARF & 2 \\
\hline
\end{tabular}

Table 3: system parameters

\begin{tabular}{|l|l|}
\hline number of subcarriers & 256 \\
\hline CR & $4 \mathrm{~dB}$ \\
\hline SNR 16QAM & $15 \mathrm{~dB}$ \\
\hline SNR 64QAM & $20 \mathrm{~dB}$ \\
\hline
\end{tabular}

\subsection{Flat Fading Channel}

This section shows the performance of the BER of OFDM signals modulated with 16 and 64QAM and in a flat fading channel when varying the SNR and the CR .Figures 8, 9 and 10 show the BER performance of a 16QAM modulation when varying the SNR from 0 to $18 \mathrm{~dB}$. The simulations in Figure 8 show the performance of the DAR and IDAR methods when no filter is applied at the transmitter. The results show that the BER performance for DAR and for IDAR is very similar, being IDAR slightly better. When the FFT filter is used at the transmitter, IDARF shows clearly better performance than DAR and IDAR. Figure 10 shows that IDARF is also better than IDAR without filter at the transmitter, keeping the BER very close to that of the no clipping case.

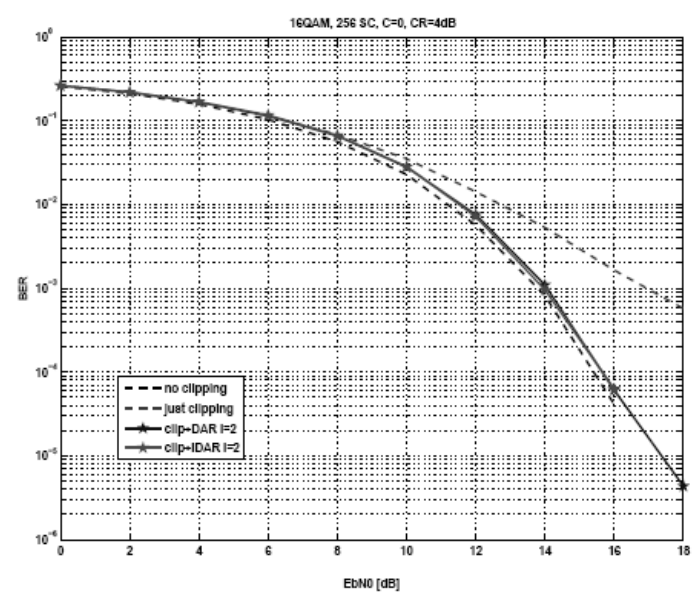

Fig 8: DAR and IDAR methods with no filter in the transmitter, 16QAM modulation in flat fading channel, $\mathrm{CR}=4$ $\mathrm{dB}, \mathrm{N}=256$ subcarriers

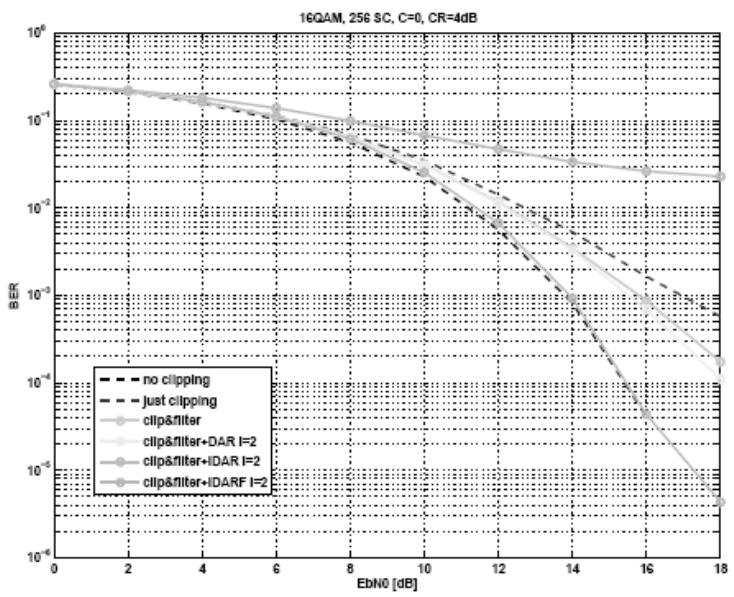

Fig 9: IDARF, DAR and IDAR methods with filter in the transmitter, 16QAMmodulation in flat fading channel, $\mathrm{CR}=4$ $\mathrm{dB}, \mathrm{N}=256$ subcarriers

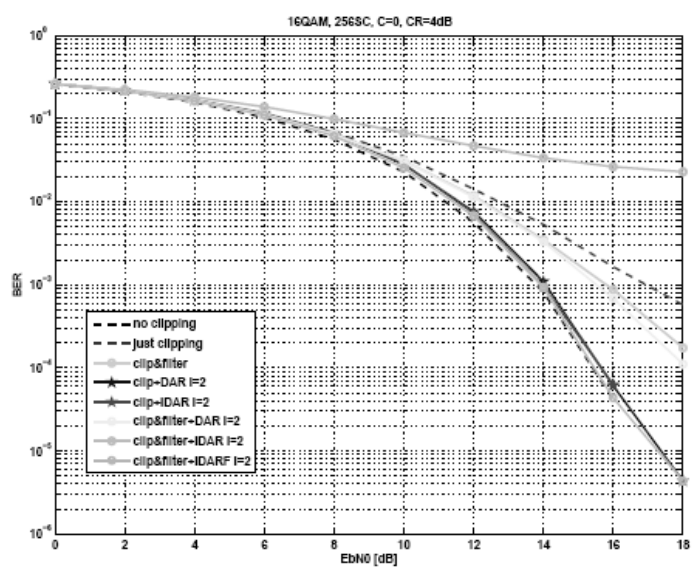

Fig 10: DAR, IDAR and IDARF methods with and without filter in the transmitter,16QAM modulation in flat fading channel, $\mathrm{CR}=4 \mathrm{~dB}, \mathrm{~N}=256$ subcarriers

Figures 11, 12 and 13 show the same simulations as the previous figures but this time holding the SNR at $15 \mathrm{~dB}$ and varying the $\mathrm{CR}$ from 2 to $14 \mathrm{dBs}$. For $\mathrm{CR}>4 \mathrm{~dB}$, the performance of DAR and IDAR in Figure 11, when no filter is applied, is approximately the same, for $\mathrm{CR}<4 \mathrm{~dB}$ performs better than DAR. When the filter is applied, Figure 12, and for $\mathrm{CR}<8 \mathrm{~dB}$ approximately, IDARF provides a significant better performance than DAR or IDAR and DAR provides no improvement compared to just clip\&filter, also the difference between IDARF and DAR or IDAR without filter in the transmitter is very small, see Figure 13. As the CR decreases, the performance of all methods degrades, being DAR without filtering at the transmitter the one that faster degrades. From a 
$\mathrm{CR}$ of $8 \mathrm{~dB}$ the performance of all methods becomes flat and similar for all them.

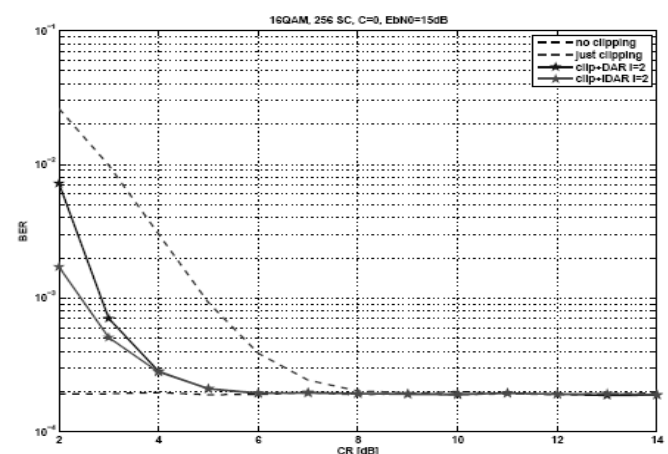

Fig11: DAR and IDAR methods with no filter in the transmitter, 16QAM modulation in flat fading channel, $\mathrm{SNR}=15 \mathrm{~dB}, \mathrm{~N}=256$ subcarriers

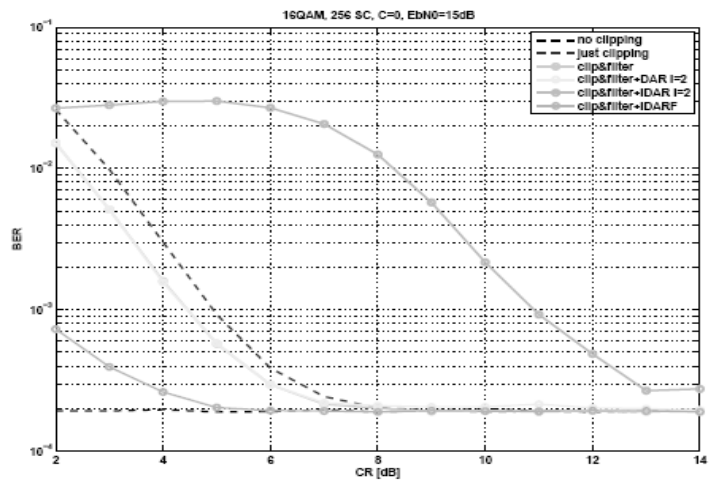

Fig 12: IDARF, DAR and IDAR methods with filter in the transmitter, 16QAM modulation in flat fading channel, $\mathrm{SNR}=15 \mathrm{~dB}, \mathrm{~N}=256$ subcarriers

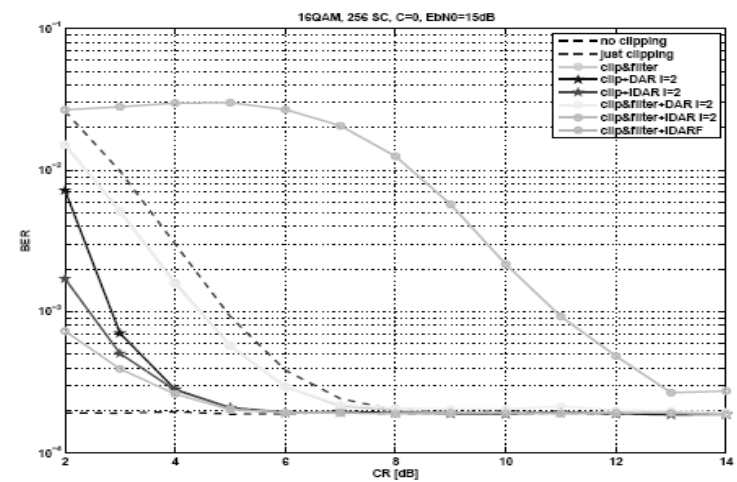

Fig13: DAR, IDAR and IDARF methods with and without filter in the transmitter, 16QAM modulation in flat fading channel, $\mathrm{SNR}=15 \mathrm{~dB}, \mathrm{~N}=256$ subcarriers
Figures 14, 15 and 16 show the BER performance of a 64QAM modulation when varying the SNR from 0 to $30 \mathrm{~dB}$ and for a clipping ratio of $4 \mathrm{~dB}$. In Figure 4.7, when no filter is applied at the transmitter, the IDAR method performs clearly better than DAR for SNR> $12 \mathrm{~dB}$ approximately, for lower SNR their performance is nearly the same. For an SNR $<20$ $\mathrm{dB}$ they behave like when no clipping is applied and after that they behave like when just clipping is applied. When the filter is used in the transmitter, Figure 4.8 , the performance of IDARF for the whole SNR range is very close to that when no clipping is applied, on the other hand, there is nearly no difference between using IDAR and using just clip\&filter. When comparing all methods in Figure 4.9, IDARF performs clearly better than DAR and IDAR without filter in the transmitter.

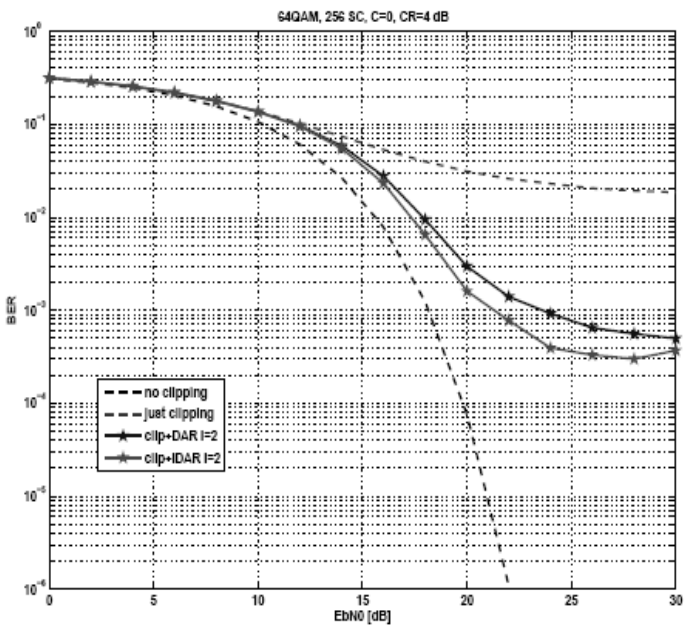

Fig 14: DAR and IDAR methods with no filter in the transmitter, 64QAM modulation in flat fading channel, $\mathrm{CR}=4$ $\mathrm{dB}, \mathrm{N}=256$ subcarriers

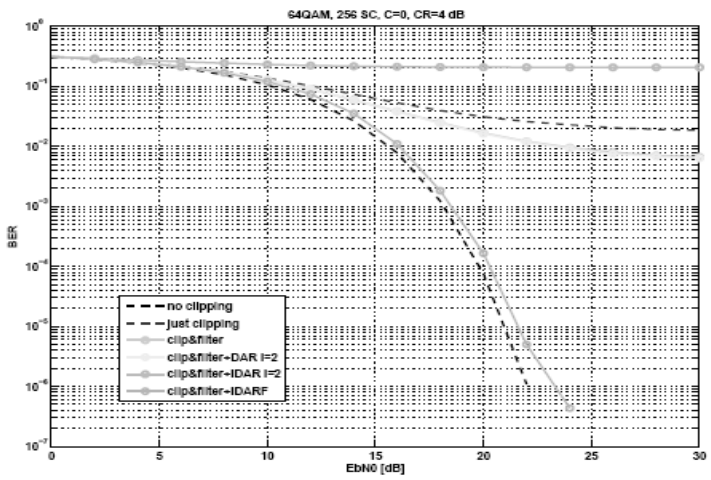

Fig 15: IDARF, DAR and IDAR methods with filter in the transmitter, 64QAMmodulationin flat fading channel, $\mathrm{CR}=4$ $\mathrm{dB}, \mathrm{N}=256$ subcarriers 


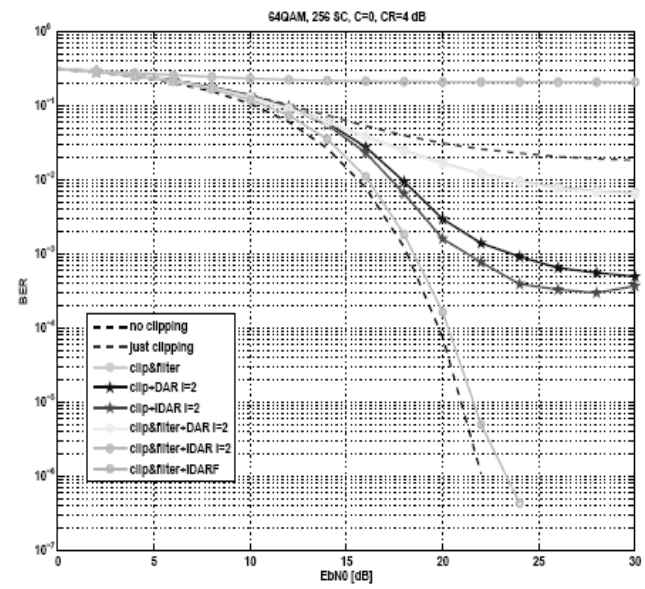

Fig 16: DAR, IDAR and IDARF methods with and without filter in the transmitter,64 QAM modulation in flat fading channel, $\mathrm{CR}=4 \mathrm{~dB}, \mathrm{~N}=256$ subcarriers

Figures 17, 18 and 19 show how those methods behave when holding the SNR at $20 \mathrm{~dB}$ and varying the CR from 2 to 14 $\mathrm{dB}$. Without filter in the transmitter, Figure 17, IDAR performs slightly better than DAR for CR $<5 \mathrm{~dB}$, after that the performance is approximately the same. With filter in the transmitter, 18, IDARF performs much better than the other methods for $\mathrm{CR}<9 \mathrm{~dB}$ and using DAR yields the same results as just clip\&filter. Regarding the whole set of methods, Figure 19, IDARF performs better than IDAR and DAR for CR $<6$ dB.

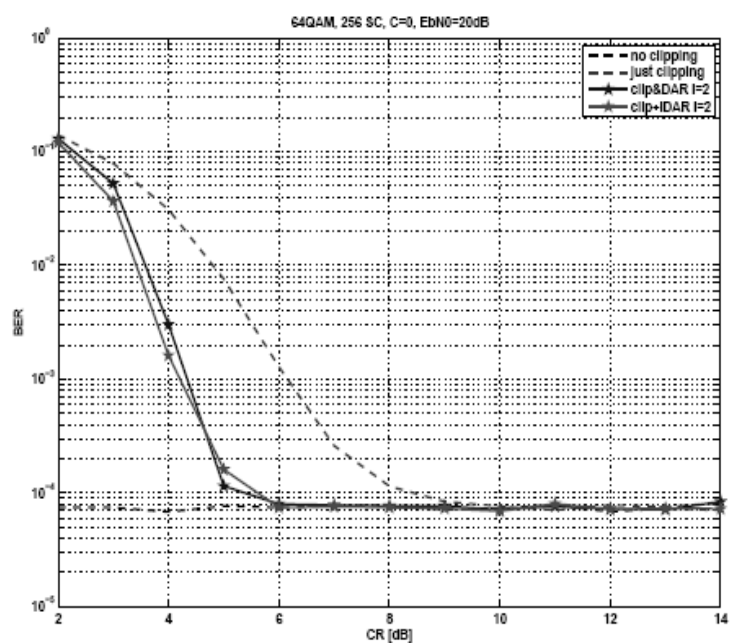

Fig 17: DAR and IDAR methods with no filter in the transmitter, 64QAM modulation in flat fading channel, $\mathrm{SNR}=20 \mathrm{~dB}, \mathrm{~N}=256$ subcarriers

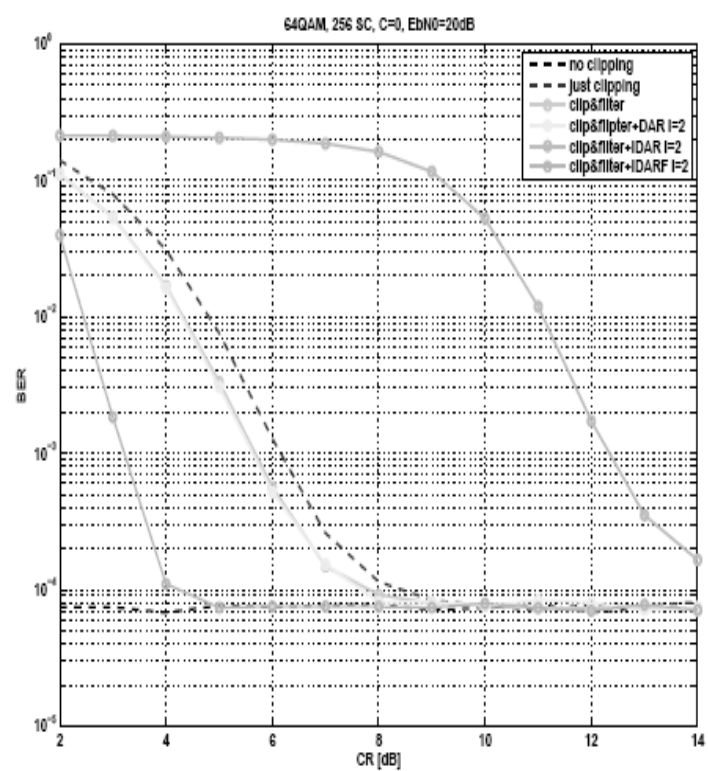

Fig 18: IDARF, DAR and IDAR methods with filter in the transmitter, 64QAM modulation in flat fading channel

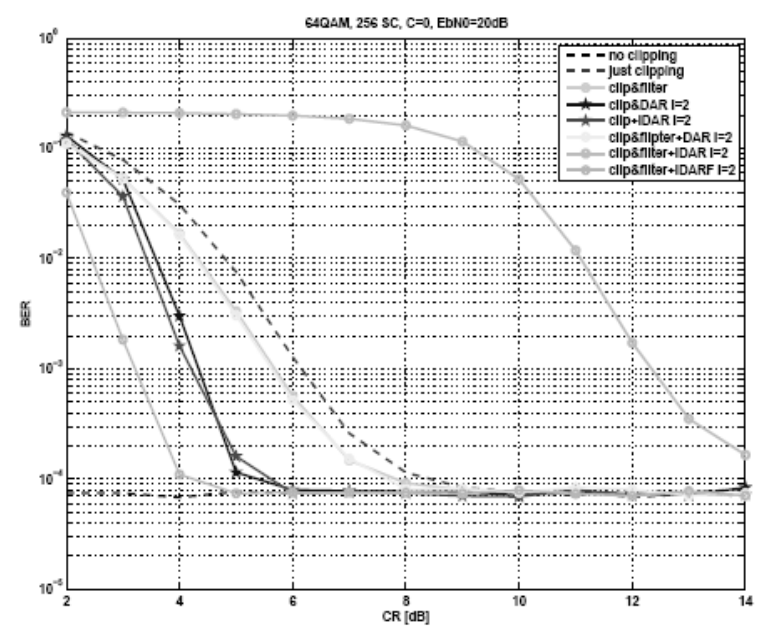

Fig 19: DAR, IDAR and IDARF methods with and without filter in the transmitter, 64QAM modulation in flat fading channel, $\mathrm{SNR}=20 \mathrm{~dB}, \mathrm{~N}=256$ subcarriers

\section{CONCLUSIONS}

When using the 16QAM modulation, DAR and IDAR have similar performance when no filter is applied at the transmitter, this performance is also very similar to that of IDARF when the FFT filter is applied at the transmitter, although IDARF performs slightly better. When the modulation used is 64QAM, IDAR performs better than DAR without filter at the transmitter and they both have similar 
behavior. IDARF, on the other hand, performs clearly better than DAR and IDAR and its behavior is closer to the case when no clipping is applied than to IDAR and DAR. When the methods are analyzed for different clipping ratios, the simulations show that when the filter is used in the transmitter and for $\mathrm{CR}<7 \mathrm{~dB}$, IDARF performs better than all the other methods, for higher CR they all perform the same. When comparing all methods without filter in the receiver, IDARF performs better than IDAR and DAR for CR $<4 \mathrm{~dB}$, for higher CR their performances are very similar. The filter is an important element in OFDM systems since it reduces the outof-band radiation and ICI caused by clipping. As seen in the simulations, when the filter is used in the transmitter, the method that better performs in the receiver is IDARF. Therefore, when clipping is applied, the better solution in order to mitigate the noise that it introduces is to use the FFT filter together with the IDARF method.

\section{REFERENCES}

[1] Jean Armstrong. "New OFDM Peak-to-average Reduction Scheme". In IEEE Vehicular Technology Conference, Rhodes, Greece, 2001

[2] Pisit Boonsrimuang, Kazuo Mori, Tawil Paungma, and Hideo Kobayashi. "Proposal of Clipping and Inter-modulation Noise Mitigation Method for OFDM Signal in Non-linear Channel". IEICE Trans. Commun., 2005.

[3]Khaled Fazel and Stefan Kaiser. Multi-Carrier and Spread Spectrum Systems. Wiley,2003.

[4]Hiroshi Harada and Ramjee Prasad. Simulation and Software Radio for Mobile Communications.Artech House, 2003.

[5]Dukhyun Kim and Gordon L. Stuber. "Clipping Noise Mitigation for OFDMby Decisionaided Reconstruction". IEEE Communication Letters, 1999.

[6]Xiaodong $\mathrm{Li}$ and Leonard J. Cimini. "Effects of Clipping and Filtering on the Performance of OFDM". IEEE Communication Letters, 1998

[7]Ye (Geoffrey) Li and Gordon L. Stuber. Orthogonal Frequency Division Multiplexing for Wireless Communications. Springer, 2006.

[8]Ramjee Prasad. OFDM for Wireless Communication Systems. Artech House, 2004

[9]Henrik Schulze and Christian Luders. Theroy and Applications of OFDM and CDMA. Wideband Wireless Communications. Wiley, 2003

[10]H. Sizun. Radio Wave Propagation for Telecommunication Applications. Springer,2003

\section{BIOGRAPHIES}

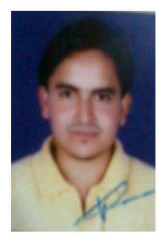

Hemant Choubey, he has received the B.E. degree in Electronics and communication engineering from Rajiv Gandhi Technical University Bhopal, in 2009. He is currently pursuing M.E degree in Digital Communication from RGTU Bhopal. 